\title{
Combinatorial Optimization of Heterogeneous Catalysts Used in the Growth of Carbon Nanotubes
}

\author{
Alan M. Cassell ${ }^{*}$, , Sunita Verma ${ }^{\ddagger}$, Lance Delzeit, M. Meyyappan, Jie $\mathrm{Han}^{\dagger}$ \\ National Aeronautics and Space Administration \\ Ames Research Center \\ Moffett Field, CA 94035 \\ ${ }^{\dagger}$ Eloret Corporation \\ ${ }^{\text {IS }}$ cience Systems and Applications Inc.
}

Libraries of liquid-phase catalyst precursor solutions were printed onto iridium-coated silicon substrates and evaluated for their effectiveness in catalyzing the growth of multi-walled carbon nanotubes (MWNTs) by chemical vapor deposition (CVD). The catalyst precursor solutions were composed of inorganic salts and a removable tri-block copolymer $(\mathrm{EO})_{20}(\mathrm{PO})_{70}(\mathrm{EO})_{20}(\mathrm{EO}=$ ethylene oxide, $\mathrm{PO}=$ propylene oxide) structure-directing agent (SDA), dissolved in ethanol/methanol mixtures. Sample libraries were quickly assayed using scanning electron microscopy after CVD growth to identify active catalysts and CVD conditions. Composition libraries and focus libraries were then constructed around the active spots identified in the discovery libraries to understand how catalyst precursor composition affects the yield, density and quality of the nanotubes. Successful implementation of combinatorial optimization methods in the development of highly active, carbon nanotube catalysts is demonstrated, as well as the identification of catalyst formulations that lead to varying densities and shapes of aligned nanotube towers.

\section{Introduction}

Starting from an empirical knowledge of required properties for active nanotube catalysts $^{1.3}$, we designed discovery libraries containing mixtures of $\mathrm{Al}, \mathrm{Si}, \mathrm{Fe}$, and $\mathrm{Co}$. Our long- 
term goal is the development of a catalyst database where formulations and reaction conditions for the desired density and/or morphology of nanotubes is created using high-throughput experimentation. The methodology described herein is widely applicable to the identification and optimization of materials for many diverse applications ${ }^{4-5}$ where liquid-based preparation and deposition is desired.

Various approaches are used to obtain catalyst materials that are active in the CVD growth of carbon nanotubes. Typically, the catalysts are composed of transition metal nanoparticles supported on the surface of materials such as silicon, silica, alumina or aluminosilicates. ${ }^{6-9}$ The catalyst composition and conditions for nanotube growth are usually determined by trial and error, a tedious and time-consuming process. As a result, little knowledge is gained using these approaches as compared to high-throughput experimentation, because often the goal is to produce carbon nanotubes in high yields. We have chosen to evaluate the catalyst activity of the heterogeneous catalyst precursors due to the ease of varying the catalyst content using solution-based preparation. The direct printing of liquid phase precursor microarrays is versatile and allows many parameters to be explored efficiently. For instance, slight variations in catalyst stoichiometry, concentration of SDA, and precursor concentration allow us to explore and tune the chemical composition and physical properties (surface area, porosity, and film shape) of the catalyst. ${ }^{10}$

\section{Experimental Procedure}

Materials and Reagents. $\mathrm{AlCl}_{3} \cdot 6 \mathrm{H}_{2} \mathrm{O}, \mathrm{CoCl}_{2} \cdot 6 \mathrm{H}_{2} \mathrm{O}, \mathrm{Fe}\left(\mathrm{NO}_{3}\right)_{3} \cdot 9 \mathrm{H}_{2} \mathrm{O}, \mathrm{SiCl}_{4}$, ethanol and methanol from Sigma-Aldrich Corporation, N-doped silicon wafers $(<100>, 1-10 \Omega-\mathrm{cm})$ from Wafer World Inc. (West Palm Beach, FL), quartz microscope slides from Chemglass Inc. (Vineland, NJ), iridium, platinum and chromium metal ( $99.99 \%$ purity) from Alfa Aesar Inc. 
argon (99.999\% Scott Specialty Gas), ethylene (99.99\% Matheson Gas Products), and P-123 triblock copolymer from BASF Inc. (Mount Olive, NJ), were used in this study.

Catalyst Solution Preparation. A typical preparation of a stock catalyst solution follows. First, $0.50 \mathrm{~g}(0.09 \mathrm{mmol})$ of Pluronic P-123 tri-block copolymer was dissolved in $15 \mathrm{~mL}$ of a $2: 1$ mixture containing ethanol and methanol. Next, $\mathrm{SiCl}_{4}(0.85 \mathrm{~mL}, 7.5 \mathrm{mmol})$ was slowly added via syringe into the tri-block copolymer/alcohol solution, and allowed to stir for 30 min at room term: :erature. Stock solutions of $\mathrm{AlCl}_{3} \cdot 6 \mathrm{H}_{2} \mathrm{O}, \mathrm{CoCl}_{2} \cdot 6 \mathrm{H}_{2} \mathrm{O}$, and $\mathrm{Fe}\left(\mathrm{NO}_{3}\right)_{3} \cdot 6 \mathrm{H}_{2} \mathrm{O}$ were prepared at the same concentration of SDA and inorganic salts. Catalyst precursor solutions were then prepared by mixing the stock solutions in varying ratios to explore active catalyst compositions (figure la). The catalyst precursor solutions were filtered through a $0.45 \mu \mathrm{m}$ polytetrafluoroethylene membranes (Osmonics Inc.) prior to printing.

Catalyst Printing. In a typical printing, the catalyst precursor solutions $(\sim 40 \mu \mathrm{L}$ of each precursor solution) were placed into the wells of a 384-well microplate (Fisher Scientific), and loaded into the robotic microarrayer (Model 417 Microarrayer, Affymetrix Inc.). "Quartz, silicon or iridium coated silicon substrates were then placed into the arrayer and the libraries were printed after calibration of the robot. Approximately $30-50 \mathrm{pL}$ of catalyst solution is transferred per printing, and typical spot diameters are roughly 150-200 $\mu \mathrm{m}$; spot diameter depends on the surface properties of the spotting solution and the substrate.

Substrate Optimization. We evaluated quartz, Si and various metal coatings on quartz and Si to optimize the substrate. We found that iridium (>30 nm) sputtered films on either quartz or $\mathrm{Si}$ are very suitable for printing of the catalysts and growth of nanotube towers. We also evaluated films of ion-sputtered $\mathrm{Pt}$ and $\mathrm{Cr}$ (chosen due to their relative inertness and stability at temperatures up to $750^{\circ} \mathrm{C}$ ). All the surfaces evaluated were effective for printing and growth, 
however the iridium films are porous (average particle size $-5 \mathrm{~nm}$, from STM analysis), which can help in the diffusion of active hydrocarbon species, ${ }^{8}$ and also aid in the dehydrogenation of hydrocarbons. $^{12}$

Chemical Vapor Deposition. The printed chips were loaded into a 1 -inch diameter tube furnace (Lindbergh Blue) and heated to $700^{\circ} \mathrm{C}$ for $4 \mathrm{~h}$ in air to render the precursor active (decomposition of the the inorganic salts and removal of the SDA). ${ }^{10}$ Ethylene flow rates between $100 \mathrm{sccm}$ and 5000 sccm (MKS Instruments mass flow controllers), and deposition temperatures between 600 ${ }^{\circ} \mathrm{C}$ and $800^{\circ} \mathrm{C}$ were investigated. Reaction time was varied between $5 \mathrm{~min}$ and $1 \mathrm{~h}$, but typical reaction time was $40 \mathrm{~min}$.

Microscopy Characterization. The reacted chips were examined using a Hitachi S-4000 field emission scanning electron microscope (SEM) operating between $15-30 \mathrm{kV}$ accelerating voltages. Up to eight chips can be loaded into the instrument at one time (150 spots), and qualitatively evaluated in less than $15 \mathrm{~min}$. More detailed examination at high magnification was done for active spots to evaluate tower density and nanotube quality. Further confirmation of nanotube quality (crystallinity) was performed using a JEOL $200 \mathrm{CX}$ transmission electron microscope operating at $200 \mathrm{kV}$ accelerating voltage.

\section{Results}

Our general approach for optimizing the nanotube catalysts is depicted in Scheme 1. First, the stock catalyst solutions are mixed in the appropriate ratios to obtain catalyst precursor mixtures that vary in stoichiometry (see Figure la for a typical discovery library). The precursor solutions are then transferred to the wells in a $386-$ well microplate $(\sim 40 \mathrm{uL}$ of catalyst precursor loaded into each well), placed into the robotic-printer, and printing commenced. Typically, twelve identical 25-member arrays were printed onto the substrate and the catalyst precursors 
were then decomposed at $700{ }^{\circ} \mathrm{C}$ overnight in air to remove the SDA, and decompose the inorganic salts into oxides. The substrates were then diced into individual chips 5 and loaded into the CVD furnace, where catalyst activity was evaluated under different fumace temperatures and flow rates of ethylene. After CVD, the arrays were placed into the SEM to qualitatively evaluate yield, density, and quality of the as-grown nanotubes.

Figure lb shows the discovery library after the CVD with ethylene (flow of $500 \mathrm{sccm}$ ) at $700^{\circ} \mathrm{C}$ for $40 \mathrm{~min}$. At $700^{\circ} \mathrm{C}$, spots $7,8,12,13,14,17,18$, and 19 show significant catalytic activity as evidenced by the height of the growing nanotube tower. When the temperature is increased to $7500^{\circ} \mathrm{C}$, most of the spots that displayed significant activity at $700{ }^{\circ} \mathrm{C}$ show even more catalytic activity as evidenced by an increase in height/volume of the nanotube tower. Furthermore, spots 1 and 2 show significant catalytic activity (although spot 1 was catalytically active, we were not able to reproduce the growth in subsequent experiments, and we are further understanding this observation). The enhancement in the tower heights at $750{ }^{\circ} \mathrm{C}$ may be due to the increased diffusion of precursors (carbon from dissociated ethylene) into the catalyst pores. However, a continuing increase in height with temperature cannot be expected at elevated temperatures due to the rapid formation of pyrolytic carbon and subsequent pore clogging. At fixed temperatures, we have also varied the flow rates to optimize the growth. At sufficiently high flow rates (>1500 sccm), spots that showed significant activity in figure $1 \mathrm{~b}$ and $1 \mathrm{c}$ exhibited reduced growth levels. High flow rates and resulting reduction in residence time, we believe, are associated with a 'precursor starved' regime. Very low flow rates and longer residence times were not useful either, which again may be due to pyrolytic products coating the catalytic surface. Upon imaging the sidewalls of the nanotube towers at high magnification, we noticed that spots 2 and 7 (figure $1 \mathrm{c}$ ) afforded the highest quality nanotubes (indicated by the alignment 
and general absence of defects occurring along the length of the nanotubes). Thus, we focused our further optimization around these catalysts.

We next constructed a focus library centered on the composition of spot 7 ( $\left.\mathrm{Si}_{3} \mathrm{FeAlCo}\right)$ from figure $1 \mathrm{c}$ to see if further enhancement of catalytic activity could be made (figure 2 ). The focus library composition is shown in figure $2 \mathrm{a}$. Using the reaction conditions from figure $1 \mathrm{c}$ $\left(750^{\circ} \mathrm{C}, 500 \mathrm{sccm}\right.$ ethylene flow rate, $\left.40 \mathrm{~min}\right)$, the catalyst activity for most spots is very significant. Especially noteworthy is row one, each tower in this row shows significant growth of nanotubes, with quality of the nanotubes comparable to those from $\mathrm{Si}_{3} \mathrm{FeAlCo}$. However, since we did not see significant improvement in the quality and density of the nanotubes produced from these catalysts (as evidenced by high magnification TEM, figure $2 e$ ), we turned our attention to optimizing the composition and printing of the catalyst precursors to understand what effects the ink has on the CVD-grown nanotube towers.

Four active catalysts were chosen from the discovery library (spots $2,7,8$, and 13 from figure Ic) to optimize the concentration of the structure-directing agent in the catalyst precursor solution. As can be seen in figure 3, as the amount of SDA in the catalyst precursor is increased, the uniformity of the towers improves. The SDA appears to have dual purposes: not only does the SDA provide the necessary pore properties required for efficient catalytic growth, ${ }^{2-3}$ it seems to help form uniformly printed spots on the surface of sputtered iridium film. When there is no SDA present in the catalyst precursor solution (figure 3, row 5), the $\mathrm{Si}_{3} \mathrm{Fe}$ catalyst exhibits little activity, while the other spots show activity, but random orientation. This can be partly explained by considering the dewetting of the printed spot on the surface of the iridium sputtered film. Previous studies have shown that thin-films can dewet the surface if the surface free energy of the film is higher than that of the underlying substrate. ${ }^{13-14}$ This leads to a breakdown 
of the film through development of holes in the film. Eventually, the film forms numerous droplets where the spot was printed upon annealing. ${ }^{15}$ However, by increasing the concentration of the $(\mathrm{EO})_{20}(\mathrm{PO})_{70}(\mathrm{EO})_{20}$, more stable films are formed, thus leading to much higher uniformity in the printed spot and the CVD-grown nanotube towers (figure 3, row 1).

To further understand the spot uniformity and its effect on the nanotubes grown from it, a concentration profile on one of the optimized catalysts for MWNT growth, $\mathrm{Si}_{3} \mathrm{Fe}\left(0.5 \mathrm{M} \mathrm{Si}_{3} \mathrm{Fe}\right.$, $5.8 \mathrm{mM} \mathrm{P}-123$ ), was performed. Figure 4 shows the effect of diluting the catalyst precursor on the spot uniformity and shape. A transition from a solid tower $\left(0.5 \mathrm{M} \mathrm{Si}_{3} \mathrm{Fe} / 5.8 \mathrm{mM} \mathrm{P-123)}\right.$ to a cylinder-shaped tower (0.1 M Si 3 Fe/1.2 mM P-123) occurs. We conclude that this is due to the film stability and that there exists a critical film thickness for dewetting that leads to the formation of the ring patterns seen in the final nanotube tower. ${ }^{16}$ However, since there are multiple components in the catalyst precursor ink, it is unclear what contribution each component has on the formation of stable films. Kind et $a l^{17}$ have shown that many factors influence the film-forming properties of gel-based catalysts, including the choice of solvent and influence of counter ions (catalyst ligands).

\section{Conclusions}

We have demonstrated the use of combinatorial optimization techniques to evaluate the quality of MWNTs produced by CVD. A number of different parameters are involved in understanding the printed spot uniformity and catalyst activity, namely, chemical composition, amount of SDA in the precursor solution and concentration of the precursor solution which are easily tunable parameters that give differing nanotube quality (crystallinity) and tower density. The parameters are efficiently tuned with high-throughput tools (microarrayer and scanning 
electron microscope), and we have demonstrated the ability to optimize the catalysts efficiently ( 750 spots analyzed per week).

In order to realize their full potential in a variety of promising applications in nanotechnology, high throughput techniques are needed to identify catalyst formulations that allow the researcher to tailor the properties of the nanotubes to their specific application. The ultimate goal of this technology is to discover formulations and synthetic methods that give the researcher control over the nanotube type, diameter, and length.

\section{Acknowledgements}

The National Cancer Institute's Unconventional Innovations Program and the NASA Ames Nanotechnology Program supported this work. BASF Inc. is gratefully acknowledged for providing the tri-block copolymers. 


\section{References and Notes}

1. Cassell, A. M.; Raymakers, J. A.; Kong, J.; Dai, H. J. Phys. Chem. B 1999, $103,6484$.

2. Cassell, A. M.; Franklin, N. R.; Tombler, T. W.; Chan, E. M.; Han, J.; Dai, H. J. Am. Chem. Soc. 1999, 121, 7975.

3. Franklin, N. R.; Dai, H. Adv. Mater. 2000, 12, 890.

4. Jandeleit, B.; Schaefer, D. J.; Powers, T. S.; Turner, H. W.; Weinberg, W. H. Angew. Chem. Int. Ed. 1999, 382494.

5. Xjang, X.-D. Annu. Rev. Mater. Sci. 1999, 29, 149.

6. Hafner, J.; Bronikowski, M.; Azamian, B.; Nikolaev, P.; Colbert, D. T.; Smalley, R. E. Chem. Phys. Lett. 1998, 296, 195.

7. Kong, J.; Soh, H. T.; Cassell, A. M.; Quate, C. F.; Dai, H. Nature 1998, 395, 878.

8. Fan, S.; Chapline, M.; Franklin, N.; Tombler, T. W.; Cassell, A. M.; Dai, H. Science 1999, $283,512$.

9. Su, M.; Li, Y.; Maynor, B.; Buldum, A.; Lu, J. P.; Liu, J. J. Phys. Chem. B. 2000, 104, 6505.

10. Yang, P.; Deng, T.; Zhao, D.; Feng, P.; Pine, D.; Chmelka, B. F.; Whitesides, G. M.; Stucky, G. D. Science 1998, 282, 2244.

11. For operational details see: http://www.affymetrix.com/products/417_arrayer.html

12. Ramaswamy, A.V.; Ratnasamy, P.; Sivisanker, S.; Leonard, A. J. Proc. $6^{\text {th }}$ Int. Congr. Catal., London 1976, paper B25.

13. Oslanec, R.; Costa, A.C.; Composto, R. J. Macromolecules 2000, 33, 5505.

14. Petrov, J. G.; Ralston, J.; Hayes, R. A. Langmuir 1999, 15, 3365.

15. Fondecave, R.; Wyart, F. B. Macromolecules 1998, 31, 9305.

16. Carré, A.; Eustache, F. Langmuir 2000, 16, 2936. 
Submitted to Langmuir as a Letter

17. Kind, H.; Bonard, J.; Forro, L.; Kern, K.; Nilsson, L.; Schlapbach, L. Langmuir 2000, 16, 6877. 



Scheme 1- Procedure for the Preparation, Growth and Evaluation of the Materials Chips. 


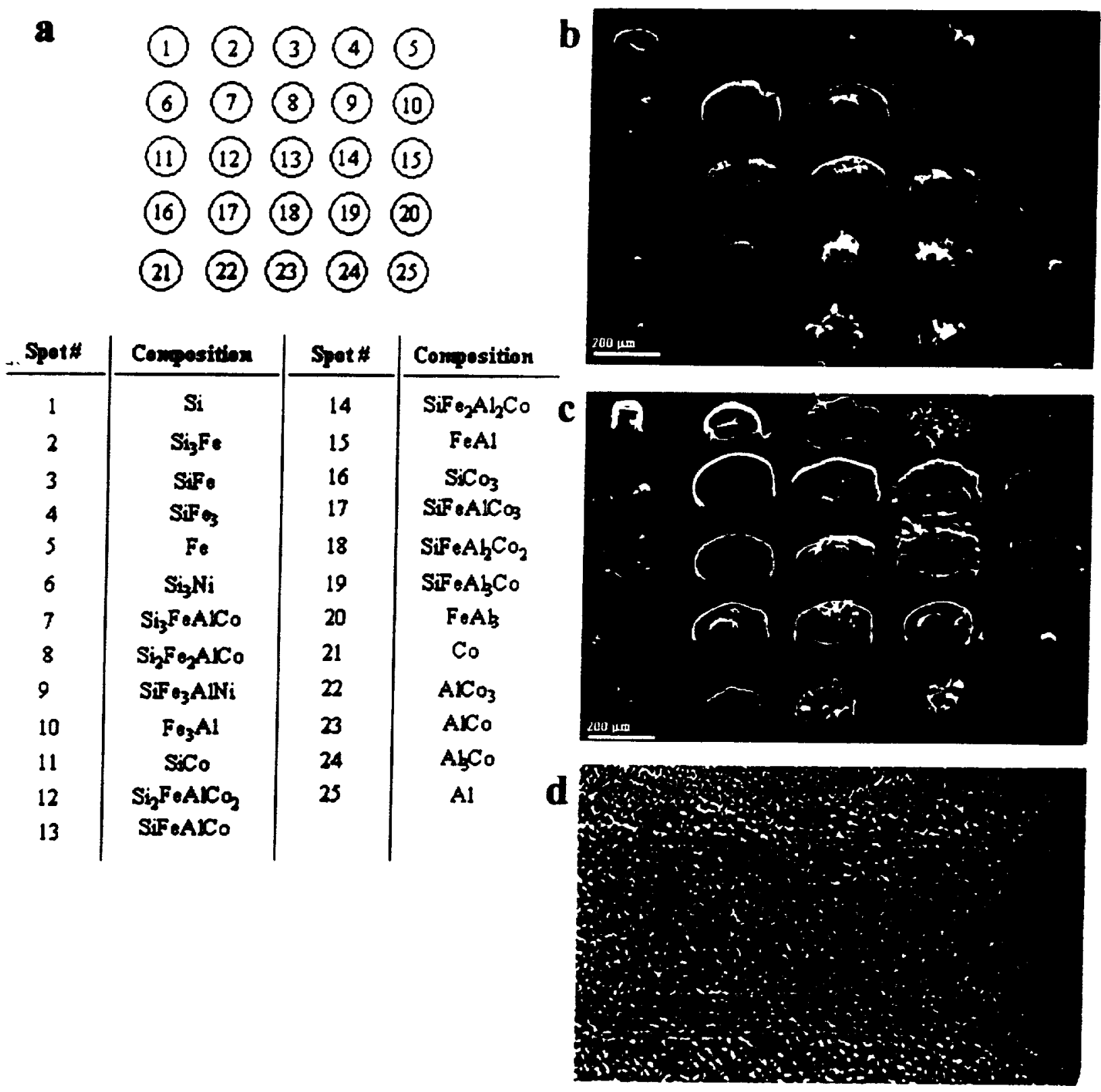

Figure 1- (a) Discovery library composition and spot locator. (b) Discovery library after CVD of ethylene at $700^{\circ} \mathrm{C}$ (500 sccm, $40 \mathrm{~min}$ deposition time). (c) Discovery librury after deposition at $750^{\circ} \mathrm{C} 500 \mathrm{sccm}, 40 \mathrm{~min}$ deposition time). Different combinations of flow rates and temperatures were evaluated (data not shown). (d) High magnification transmission electron microscope image of a nanotube from spot 7. 




Figure 2- (a) Focus library composition centered near the composition of spot 7 ( $\mathrm{Si}_{3} \mathrm{FeAlCo}$ ). (b) Focus library after after deposition at $750^{\circ} \mathrm{C}(500 \mathrm{sccm}, 40 \mathrm{~min}$ deposition time). (c) High magnification image of nanotubes from spot 2 ( $\mathrm{Si}_{3} \mathrm{Fe}$ catalyst shows similar nanotube density and quality) nanotube diameters range from $15-30 \mathrm{~nm}$. (d) High magnification image of nanotubes from spot 24 , showing lower nucleation density, and thus more defective nanotubes. 


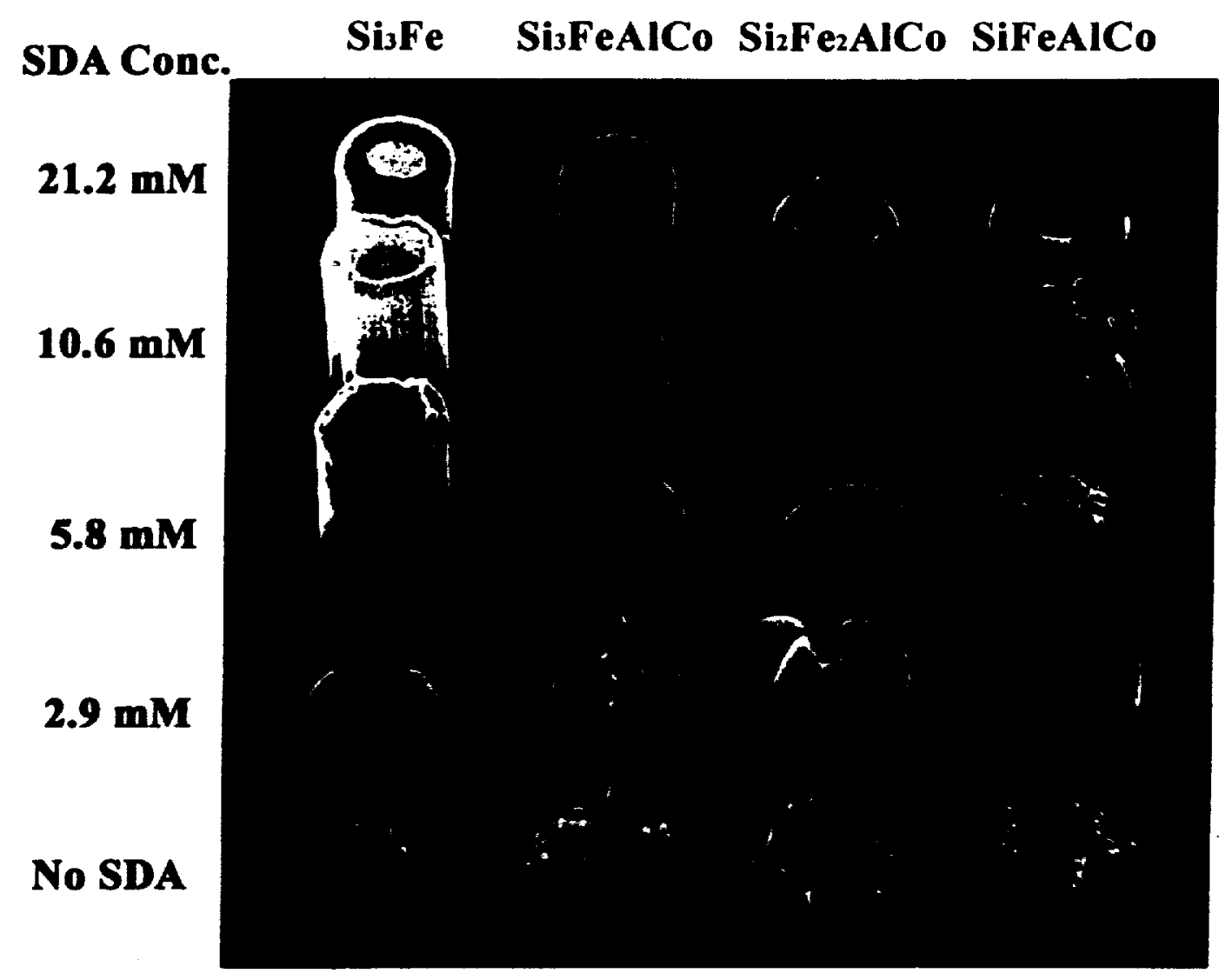

Figure 3. Structure directing agent concentration effect on spot uniformity for candidate catalysts. 




Figure 4- (a) Concentration profile for one of the optimized catalysts $\left(\mathrm{Si}_{3} \mathrm{Fe}\right)$. (b) Barrel-shaped tower from $0.2 \mathrm{M}$ catalyst precursor, and (c) solid tower from $0.5 \mathrm{M}$ catalyst precursor. 
